\title{
Concomitant Endoscopic Radiofrequency Ablation and Laparoscopic Reflux Operative Results in More Effective and Efficient Treatment of Barrett Esophagus
}

\author{
Trudie A Goers, MD, Pedro Leão, MD, Maria A Cassera, BS, Christy M Dunst, MD, FACS, \\ Lee L Swanström, MD, FACS
}
BACKGROUND: Barrett esophagus (BE) caused by gastroesophageal reflux disease can lead to esophageal cancer. The success of endoscopic treatments with BE eradication depends on esophageal anatomy and post-treatment acid exposure.

STUDY DESIGN: Between January 2008 and December 2009, 10 patients were selected for combination treatment of BE using laparoscopic anti-reflux surgery and endoscopic radiofrequency ablation. Retrospective review of preoperative, procedural, and postoperative data was performed.

RESULTS: $\quad$ Seven study patients had a pathologic diagnosis of nondysplastic BE and 3 patients had a diagnosis of low-grade dysplasia. Average length of BE lesions was $6.4 \pm 4.8 \mathrm{~cm}$. Procedure time averaged $154.4 \pm 46.4$ minutes. At the time of surgery, the mean number of ablations performed was $4.39 \pm 1.99$. Six patients were noted to have major hiatal hernias requiring reduction. Five patients $(80 \%)$ had $100 \%$ resolution of their BE at their first postoperative endoscopy. The remaining 3 patients had a $\geq 50 \%$ resolution and underwent subsequent endoscopic ablation. Symptomatic results revealed that 4 patients had substantial dysphagia to solids and other symptoms were minimal. Two patients were noted to have complications related to the ablative treatments. One stricture and 1 perforation were observed.

CONCLUSIONS: Endoscopic radiofrequency ablation of $\mathrm{BE}$ at the time of laparoscopic fundoplication is feasible and can effectively treat BE lesions. A single combined treatment can result in fewer overall procedures performed to obtain BE eradication. (J Am Coll Surg 2011;213:486-492. (C) 2011 by the American College of Surgeons)

Esophageal cancer has the fastest growing incidence rate of all cancers in the United States and Western Europe, increasing $400 \%$ during the past 35 years. ${ }^{1-4}$ Barrett esophagus $(\mathrm{BE})$ represents a marker for the potential development of esophageal adenocarcinoma. This condition develops when gastroesophageal reflux damages the distal esophageal squamous mucosa and the resulting injury heals by a metaplastic process. ${ }^{4,5} \mathrm{BE}$ can progress in a stepwise fashion from intestinal metaplasia to low-grade dysplasia to high-

Disclosure Information: Authors have nothing to disclose. Timothy J Eberlein, Editor-in-Chief, has nothing to disclose.

Abstract presented at Digestive Disease Week, New Orleans, LA, May 2010.

Received November 3, 2010; Revised June 15, 2011; Accepted June 15, 2011. From the GI/Minimally Invasive Surgery Division, The Oregon Clinic, Portland, OR (Goers, Cassera, Dunst, Swanström) and Department of Surgery, Hospital de Braga, Braga, Portugal (Leão).

Correspondence address: Lee L Swanström, MD, FACS, GI/Minimally Invasive Surgery Division, Oregon Health Sciences University, 1040 NW $22^{\text {nd }}$ Ave, Suite 560, Portland, OR 97210. email: 1swanstrom@orclinic.com grade dysplasia to carcinoma in situ and, ultimately, invasive adenocarcinoma. The mechanisms of this progression are not completely understood.

With current understanding of BE's relationship to gastroesophageal reflux disease and its risk of cancer, we believe that treatment of dysplastic BE should be 2-fold, ie, attention to the dysplastic mucosa and effective treatment of chronic gastric reflux exposure that leads to such changes.

Radiofrequency ablation (RFA) has been safely and effectively applied to $\mathrm{BE}$ with and without dysplastic changes. ${ }^{6-9}$ It usually requires 2 to 3 ablation sessions to achieve complete eradication of BE. However, anatomic distortion of the esophagus from large hiatal hernias, strictures, esophageal dilation or tortuosity, shortenedesophagus, or, possibly, a previous fundoplication (Fig. 1), can make it more difficult to achieve effective ablation. ${ }^{10-12}$ In these scenarios, RFA of $\mathrm{BE}$ can require multiple attempts or be impossible to accomplish. 


\section{Abbreviations and Acronyms}

ARS $=$ anti-reflux surgery

$\mathrm{BE}=$ Barrett esophagus

$\mathrm{RFA}=$ radiofrequency ablation

As the patient's underlying genetic predisposition to develop $\mathrm{BE}$ remains unchanged, prevention of reflux seems mandatory to prevent recurrence. Optimal control of reflux after ablation, however, has not been defined. Current options include lifetime high-dose acid suppression or antireflux surgery (ARS). The role of ARS certainly has theoretical appeal because it provides an absolute barrier to all gastric contents and there is ample evidence that acid alone is not the causative agent of $\mathrm{BE}$ or dysplasia. If one accepts the idea of adding an ARS to BE ablation, the question of the timing of the 2 procedures arises. For example, although ARS before ablation can straighten the esophagus and cure esophagitis and strictures, the fundoplication itself can interfere with effective RFA by obscuring the landmarks of the gastroesophageal junction or making access to the distal-most segments of the metaplasia more difficult. Performing ARS after an ablation can also be more difficult because of transmural inflammatory changes, or complete ablation can never be possible because of the anatomical distortion of the esophagus (angulation, dilatation, shortening) related to hiatal herniation. ${ }^{13}$

We hypothesize that performing endoscopic RFA of BE at the time of ARS would be a safe and effective method that can reduce the number of treatments needed to eradicate the metaplasia by reducing the hernia and straightening the esophagus, as well as providing the best chance of preventing future $\mathrm{BE}$ recurrence.

\section{METHODS}

This study involved the retrospective review of patient charts and information that was prospectively collected for an IRB-approved data registry containing patients who had endoscopic balloon-based esophageal RFA. All procedures were performed between January 2008 and December 2009.

\section{Patients}

Two groups of patients were included. The first included patients who were scheduled for ARS because of either failure or dislike of chronic medical therapy and who had endoscopic and histologic presence of BE with no or lowgrade dysplasia. Patients with high-grade dysplasia were excluded from selection in this group, as the presence of a

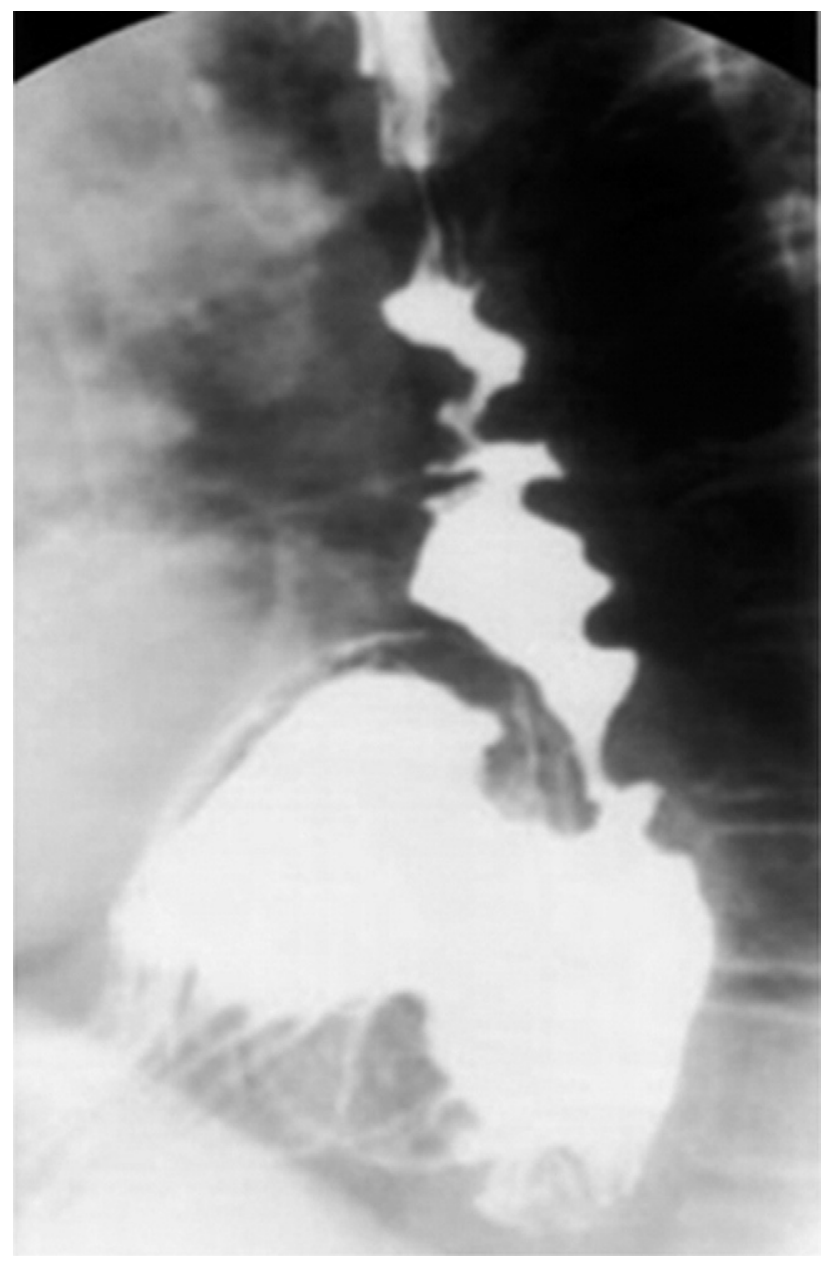

Figure 1. Contrast study of patient with long-standing gastroesophageal reflux disease, paraesophageal hernia, and chronic esophageal mucosal scarring.

fundoplication might compromise future oncologic resection and reconstruction if needed.

The second group of patients included those who originally had biopsy-proven BE with either low- or high-grade dysplasia and who were considered failures to ablate $(>3$ attempts) because of anatomic distortion of the esophagus from dilation, tortuosity, or hiatal hernias; and those who currently had either nondysplastic or low-grade dysplasia on biopsy. Likewise, patients with persistent high-grade dysplasia were excluded and were instead considered for minimally invasive esophagectomy.

\section{Presenting and procedural data}

Demographic data were prospectively collected including age, sex, and medical history. Histopathology in the absence of esophagitis, 24-hour $\mathrm{pH}$, and esophageal manometric testing were recorded in an Access-based (Mi- 


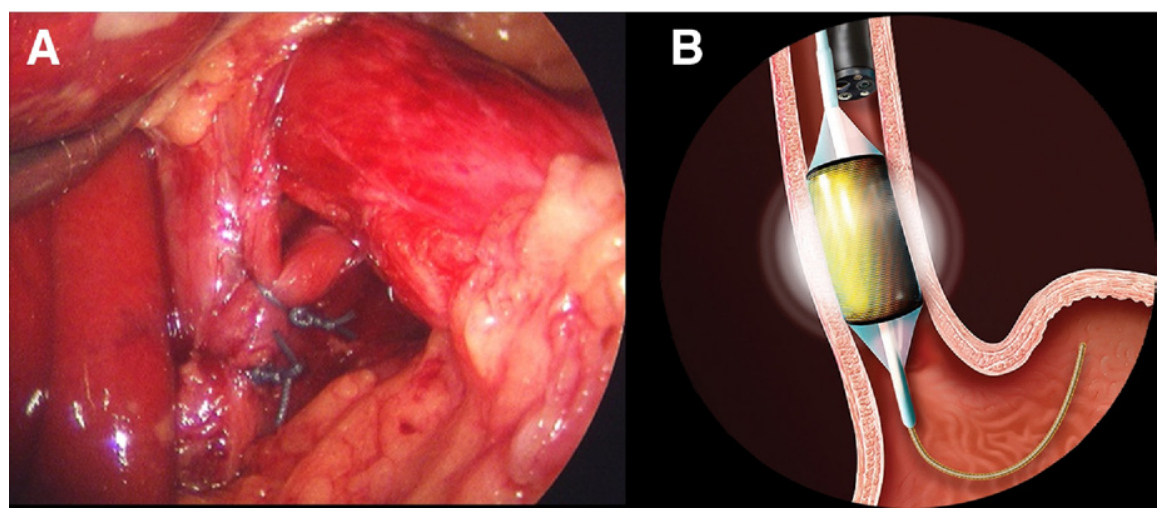

Figure 2. (A) Extensive type II mediastinal dissection allows straightening of the distal esophagus and the gastroesophageal junction rests within the abdomen. (B) The radiofrequency balloon electrode can contact esophageal mucosa.

crosoft) database. Baseline BE measurements were taken from the first endoscopy. Endoscopy was performed using white light and narrow-band imaging. The endoscopic biopsy protocol included 4 quadrant biopsies taken at $2-\mathrm{cm}$ intervals and all nodular areas were removed with endoscopic submucosal resection (EMR). Dysplasia was recorded based only on biopsies taken in the absence of acute inflammation. Presence, type, and size of hiatal hernia were also recorded. At the time of surgical procedure, information about the length of ablation, type of electrode, and details of treatment were recorded from the operative report.

\section{Surgical procedure}

All procedures were done under general anesthesia in the supine position using 5 laparoscopic ports. Mediastinal dissections were performed with the end point of the gastroesophageal junction lying $3 \mathrm{~cm}$ below the hiatus (Fig. 2). After mobilization of the distal esophagus and stomach, the esophagus was sized with a sizing balloon and an appropriate-sized RFA ablation balloon $\left(\mathrm{HALO}^{360}\right.$; Barrx Medical) was selected by a second surgeon performing endoscopy. After irrigating the esophagus with $1 \%$ $\mathrm{N}$-acetylcysteine to remove mucous, the balloon ablation catheter was inserted over a guidewire and ablation performed as the laparoscopic surgeon applied careful traction to straighten and align the esophagus to maximize contact of the ablation balloon. Two cycles of ablations were performed at either $10 \mathrm{~J}$ (no dysplasia) or $12 \mathrm{~J}$ (history of dysplasia). Two patients with more localized and limited disease had ablation with a endoscope-mounted electrode $\left(\mathrm{HALO}^{90}\right.$; Barrx Medical) instead of the balloon electrode, but using the same ablation protocol. Once the ablation was completed, repair of any hiatal hernia was performed and a tailored fundoplication was performed.

\section{Postoperative care}

All patients were admitted overnight and observed. Patients were placed on proton pump inhibitors and were given liquid pain medication as needed. Those who had concomitant performance of a paraesophageal hernia were kept nothing per os overnight and underwent esophagogastric radiographic study with water-soluble contrast on the first postoperative morning. If no leak was detected, patients were started on a liquid diet. Those with minimal hiatal hernias were started on a liquid diet the night of surgery. All patients were instructed to continue the liquid diet for 2 days, followed by a pureed diet for 2 weeks. The patients were instructed to take a single-dose proton pump inhibitor for the first 3 weeks after surgery to protect the denuded distal esophagus.

\section{Outcome measurements}

A standardized gastrointestinal symptom assessment tool was administered at each visit. Patients were followed up in clinic at 2 weeks, 3 months, and 6 months and then yearly. A focused physical examination was performed and any complications or side effects from the surgery were recorded. Patients who had any severe symptoms at any visit underwent appropriate studies and treatment at that visit.

Routine follow-up endoscopy was performed 2 to 3 months after the combination procedure to assess completeness of the ablation. Esophageal mucosa was examined using white light and narrow-band imaging. Subjective mapping of residual BE lesions' length and circumferential vs. focal nature were recorded in the endoscopic report. Four quadrant biopsies were repeated at 2-cm intervals of both normal-appearing mucosa and columnar mucosa using endoscopic jumbo forceps and were sent in formalin for pathology. Percentage of BE resolution was estimated by the endoscopist based on comparison with preoperative 
Table 1. Demographic and Preoperative Data of Study Patients

\begin{tabular}{lc}
\hline Age, $y$, mean \pm SD & $58 \pm 16.6$ \\
\hline Body mass index, mean \pm SD & $34 \pm 9$ \\
\hline Male sex, $\mathrm{n}(\%)$ & $7(70)$ \\
\hline ASA, n (\%) & $1(10)$ \\
\hline 1 & $9(90)$ \\
\hline 2 & $7(70)$ \\
\hline Histology, n (\%) & $3(30)$ \\
\hline Nondysplastic & $10(100)$ \\
\hline Low-grade dysplasia & $1(10)$ \\
\hline GERD & $3(30)$ \\
\hline Esophagitis & $7(4-6)$ \\
\hline Preprocedure ablations, $\mathrm{n}(\%)$ & \\
\hline Mone &
\end{tabular}

ASA, American Society of Anesthesiologists; GERD, gastroesophageal reflux disease.

photos and detailed endoscopy notes. Then, at 6 months, the integrity of the fundoplication was determined per our usual protocol using endoscopy, high-resolution manometry, and 24-hour $\mathrm{pH}$ studies. A competent fundoplication was defined as an intact wrap on endoscopic retroflection (Hill grade 1) and/or normal acid exposure by 24-hour $\mathrm{pH}$ tests (DeMeester score <14.7). If BE was present at the 3or 6-month visit, the patient had an additional RFA at that time.

\section{Data analysis}

Data that was collected was stored in an IRB-approved database that was developed and maintained by the principal investigator. The means of all of the continuous variables were compared using appropriate parametric or nonparametric tests. Statistical analysis was performed using Predictive Analytics Software (version 18.0; SPSS, Inc).

\section{RESULTS}

During the 24-month data collection period, 78 patients with $\mathrm{BE}$ underwent treatment, of these, 15 patients met the inclusion criteria to undergo concomitant endoscopic RFA and laparoscopic fundoplication for the simultaneous treatment of $\mathrm{BE}$ and gastroesophageal reflux disease. Of these, 1 patient requested esophagectomy, 1 patient rejected the follow-up protocol, and 3 did not want a fundoplication. Therefore, 10 patients agreed to the combined procedure.

Patients varied in age from 23 to 80 years old (Table 1). Similarly, American Society of Anesthesiologists scores were all 2, with the exception of a 23-year-old patient who was scored as a 1 . The average body mass index of the group
Table 2. Procedural Data of Study Patients

\begin{tabular}{|c|c|}
\hline Length of procedure, min, mean $\pm S D$ & $154.4 \pm 46.41$ \\
\hline Barrett's esophagus length, $\mathrm{cm}$, mean $\pm \mathrm{SD}$ & $6.4 \pm 4.78$ \\
\hline $\begin{array}{l}\text { Ablations performed during initial procedure, } n, \\
\text { mean } \pm S D\end{array}$ & $4.39 \pm 1.99$ \\
\hline \multicolumn{2}{|l|}{ Fundoplication, n (\%) } \\
\hline Nissen & $9(90)$ \\
\hline Toupet & $1(10)$ \\
\hline Presence of large hiatal hernia, $\mathrm{n}(\%)$ & $6(60)$ \\
\hline Circumferential lesions/360 balloon used, n (\%) & $8(80)$ \\
\hline \multicolumn{2}{|l|}{ Balloon size, $\mathrm{mm}, \mathrm{n}(\%)$} \\
\hline 25 & $5(50)$ \\
\hline 28 & $2(20)$ \\
\hline 31 & $3(30)$ \\
\hline Focal lesions/HALO ${ }^{90}$ used, n (\%) & $3(30)$ \\
\hline \multicolumn{2}{|l|}{ Energy, J, n (\%) } \\
\hline 10 & $6(60)$ \\
\hline 12 & $4(40)$ \\
\hline
\end{tabular}

was in the category of obese $(34 \pm 9)$, and no patient was considered to be normal (interquartile range 26.1-38.0).

At the time of the study, all patients had biopsyconfirmed BE. Seven study patients had a pathologic diagnosis of nondysplastic $\mathrm{BE}$ and 3 patients had a diagnosis of low-grade dysplasia. All 10 patients had abnormal 24-hour $\mathrm{pH}$ testing. All patients had high-resolution manometry and 1 patient had a profound primary esophageal dysmotility. Only 1 patient had active esophagitis at the time of diagnosis. Seven of the patients were considered to have failed RFA because of BE persistence despite multiple ablation attempts ( 4 to 7 attempts). The other 3 were de novo patients with long-segment BE (2 with low-grade dysplasia, 1 without) who were seeking ARS for symptom control because of failure of medical management.

\section{Procedural data}

The combined procedure time averaged $154.4 \pm 46.4$ minutes (Table 2). At the time of surgery, 6 patients were noted to have major hiatal hernias (type III) requiring reduction and crural reconstruction. Nine patients underwent 360-degree fundoplication and the patient with poor esophageal motility had a 270 -degree posterior fundoplication.

The average length of BE lesions was $6.4 \pm 4.8 \mathrm{~cm}$ (Table 2). Eighty percent required the 360-degree balloon electrode for circumferential disease. The 90-degree HALO electrode was used in $20 \%$ for more focused energy application. One patient had both circumferential and focal islands of disease and required the use of both the 360 degree and 90-degree electrodes. Of the 3 patients with dysplasia, all had energy applied at $12 \mathrm{~J} / \mathrm{cm}^{2} 3$ with the 


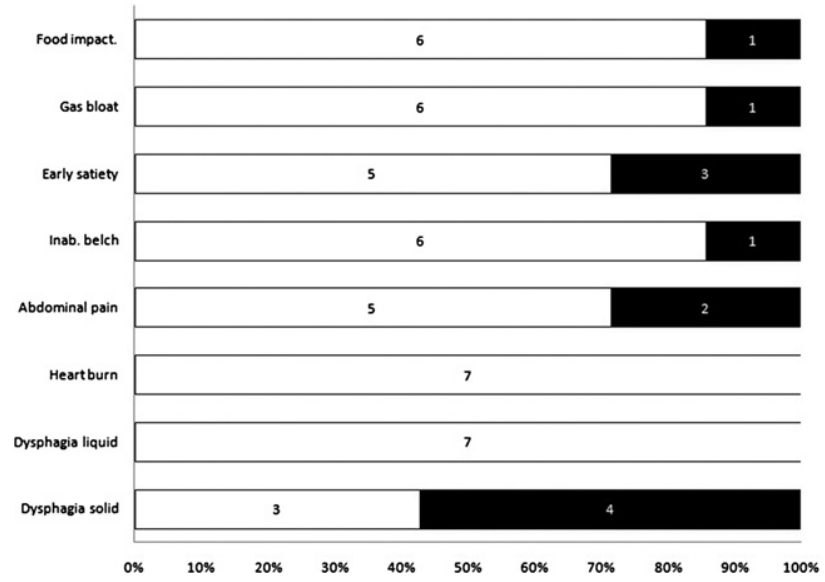

Figure 3. Postoperative patient symptomatic evaluation at 2 to 4 weeks. White bar, absent; black bar, present.

360-degree device. The diameter of the 360-degree balloon electrode used varied. Fifty percent of patients required the $25-\mathrm{mm}$ balloon. However, other patients had larger esophageal diameters and the $28-\mathrm{mm}(\mathrm{n}=2)$ or $31-\mathrm{mm}(\mathrm{n}=3)$ balloon electrodes were used.

There were no surgical or endoscopic complications and blood loss was $<50 \mathrm{~mL}$ in all cases. Eight patients were discharged home on postoperative day 1 and 2 at 48 hours because of transportation issues. There were no readmissions or acute perioperative problems.

\section{Symptomatic evaluation}

Patients were seen between 2 and 4 weeks postoperatively for acute recovery data, including pain control, diet progression, and other subjective data. Results of the symptom questionnaire are shown in Figure 3.

\section{BE resolution}

Long-term follow-up ranged from 7 months to 28 months (mean 17 months). All patients completed their 6-month comprehensive evaluation, 8 completed their 1 -year evaluation, and 4 completed a 24-month follow-up.

All patients were free of $\mathrm{BE}$ at time of last follow-up. One had biopsies with columnar epithelium, but no intestinal metaplasia. Six patients $(60 \%)$ had $100 \%$ resolution of their BE after 1 intraoperative ablative treatment performed at the time of their fundoplication (Table 3). This included 4 patients who had failed previous multiple attempts at ablation. None of the remaining BE patients had dysplasia. The remaining 4 patients had a $\geq 50 \%$ resolution and underwent endoscopic ablation. At their second follow-up endoscopy, 3 patients were found to have residual BE, however, their overall disease burden was less. A third ablation succeeded in complete control, although 1
Table 3. Endoscopic Evaluation Results of Barrett Esophagus Resolution Status Post Combined Therapy

\begin{tabular}{ccclc}
\hline $\begin{array}{l}\text { Patient } \\
\text { no. }\end{array}$ & $\begin{array}{c}\text { Days } \\
\text { post- } \\
\text { operation }\end{array}$ & $\begin{array}{c}\% \\
\text { Resolution }\end{array}$ & Complication & $\begin{array}{c}\text { Hill grade } \\
\text { fundoplication }\end{array}$ \\
\hline 1 & 205 & 100 & No & 1 \\
\hline 2 & 48 & 100 & Yes, stricture* & 1 \\
\hline 3 & 90 & 50 & No & 1 \\
\hline & 159 & 75 & No & 1 \\
\hline & 220 & 100 & No & 1 \\
\hline 4 & 78 & 85 & No & 1 \\
\hline & 166 & 95 & No & 1 \\
\hline 5 & 249 & 100 & No & 1 \\
\hline 6 & 202 & 100 & Yes, perforation & 1 \\
\hline 7 & 186 & 100 & No & No \\
\hline 8 & 187 & 50 & No & 1 \\
\hline & 255 & 100 & No & 1 \\
\hline 9 & 190 & 100 & No & 1 \\
\hline 10 & 60 & 80 & No & 1 \\
\hline & 376 & $100^{*}$ & No & \\
\hline
\end{tabular}

${ }^{*}$ Columnar epithelium without intestinal metaplasia.

patient continued to have columnar epithelium with no intestinal metaplasia at 24-month follow-up.

Two patients had major complications related to the ablation treatments. One patient had a soft stricture noted at their first diagnostic endoscopy performed on postoperative day 48 for mild solid-food dysphagia. A second patient was evaluated at 6 weeks postoperatively because of a report of a food impaction. This patient was evaluated with upper endoscopy and was found to have a $1.5-\mathrm{cm}$ perforation within the proximal RFA field.

There were no particular postoperative complications attributable to the fundoplication, although patients frequently noted common side effects, such as early satiety, bloating, and flatulence. At longest follow-up, no patients had reflux complaints and 1 patient reported heartburn. Three patients were on peptic medications. All fundoplications were intact (Hill grade I) on last endoscopy. Eight patients had postoperative manometry and $\mathrm{pH}$ studies and all results were within the normal range, including the 1 patient with heartburn and all 3 patients on proton pump inhibitors postoperatively.

\section{DISCUSSION}

Treatment of BE with endoscopic RFA is a relatively new concept. The technique so far has been reported to be both effective and well-tolerated. ${ }^{10,11}$ Exact indications for its use, however, have yet to be worked out completely and the long-term efficacy of endoscopic ablations remains unknown. The cost-effectiveness of this treatment is also a 
controversial subject and both the efficacy and costeffectiveness of the procedure rely primarily on the success rate of $\mathrm{BE}$ eradication; how many treatments it takes to achieve eradication; and how long the $\mathrm{BE}$ stays ablated. The literature describes a $>95 \%$ eradication rate after an average of 2 to 6 treatments. ${ }^{6,12,14}$ Anecdotally, however, most practitioners have encountered a substantial number of patients who seemingly cannot be ablated despite multiple treatment sessions. At our high-volume center $(>250$ RFA procedures), we have had 1 patient referred to us after 16 ablation attempts and another after 12. Even with our experience, we have had occasions where as many as 5 ablations failed to achieve clearance. Considering the standard protocol of treatment with RFA, reassessment 2 to 3 months later and retreatment if there is residual disease, interspersed with occasional endoscopies for biopsy evaluation, it is easy to understand the enormous health care burden that multiple treatment sessions impose on the patient, practitioner, and health system.

In our experience, multiple sessions of endoscopic RFA were most often needed for patients who had anatomic distortion of their distal esophagus because of hiatal hernias, chronic peptic scarring, or esophageal dilation and tortuosity. We therefore theorized that laparoscopically dissecting and freeing the distal esophagus would allow us to straighten it and make its lumen more uniform. This, in turn, would allow us to better visualize endoscopically the esophageal mucosa, more accurately calibrate balloon sizes, and more effectively deliver radiofrequency energy to the $\mathrm{BE}$ lesions. In fact, our study shows that even in patients who had failed multiple treatments, the majority of the study patients had complete resolution of $\mathrm{BE}$ after 1 intraoperative ablation session using this intraoperative strategy.

We also like the idea of leaving a patient with a mechanical reflux barrier at the completion of their myotomy. It is presumed, but not known at this time, that the neosquamous mucosa after $\mathrm{BE}$ ablation will have the same genetics as the original esophagus and, therefore, be at high (perhaps inevitable) risk of reconverting to $\mathrm{BE}$ and possible dysplasia unless something additional is done to prevent it. An intact fundoplication is well known to give patients a supraphysiologic reflux barrier that probably would have an impact on the genetic predisposition to metaplastic transformation. Whether medical therapy would have the same effect is controversial. There is much evidence that would support BE progression, even in the face of medical treatment or perhaps because of it. We do plan to follow this patient cohort, as well as our medically treated patients, for the long-term to document the rate of $\mathrm{BE}$ recurrence in both groups.

Although the combined procedure required more oper- ative time (154 minutes) than an average fundoplication case, the overall cost savings to the patient, physician, and health care system as a whole could be substantial. If $\mathrm{BE}$ could be eliminated in $20 \%$ of chronic reflux patients using $20 \%$ fewer resources and personnel, the longer operative time and initially higher procedure costs would yield large cost savings overall.

Poor understanding of the genetic pathways of BE neogenesis results in our incomplete knowledge of how to best treat histologic subtypes of BE. ${ }^{15,16}$ In this study, 2 patients (patients 3 and 4) required $>2$ ablations, even with intraoperative mobilization. Both of these patients' initial pathology was nondysplastic BE. There were no significant differences in the endoscopic RFA portion of these 2 cases. Despite no notable difference from the other study patients who achieved complete ablation in 1 session, these 2 patients had persistent BE. Besides technical error, a possible explanation for the different response to treatment could be the genetic profile of the patients. It might be that they possess proto-oncogenes or RNA triggers that make their $\mathrm{BE}$ cells more resistant to radiofrequency energy, or make regenerating neomucosa more likely to go down the $\mathrm{BE}$ pathway. A better understanding of genetic predisposition and therapeutic sensitivities is certainly needed in $\mathrm{BE}$ patients.

The complication rate for our study was 20\% (2 patients), indicating that the combined procedure is fairly well tolerated. Individually, laparoscopic ARS and endoscopic RFA treatment are acceptably safe and have a complication rate $<10 \%$. The patient in whom the minor stricture developed was minimally symptomatic, underwent a single dilation, and has not had any residual symptoms. The other complication, necrotic perforation, is more worrisome. The national BARRX registry had not previously reported any perforations related to its device and no other authors have described this phenomenon. ${ }^{6,9,17}$ Our patient in whom a perforation developed was an octogenarian who had a $13-\mathrm{cm}$ length of $\mathrm{BE}$. His procedure was uneventful and he recovered without incident. He presented 6 weeks later with a report of progressive dysphagia and food impaction. Endoscopy revealed a 1.5 -cm necrotic perforation $6 \mathrm{~cm}$ above the lower esophageal sphincter (LES). A contrast study confirmed a contained, self-draining perforation. This patient was treated conservatively and discharged to home on a liquid diet and oral antibiotics. After 2 months, endoscopy showed complete healing of his esophagus, no $\mathrm{BE}$, and an intact fundoplication. He was advanced to a regular diet and has subsequently done well. We now treat long segments in a staged fashion. The overall tissue quality in these patients with long-standing disease is generally poor, distal esophageal mobilization can be diffi- 
cult and result in tissue damage and thinning of the esophageal wall. With this, radiofrequency energy application overall distance can compromise tissue microvasculature and result in easier necrosis. Because of these concerns, we currently limit our ablations to lesions $\leq 5 \mathrm{~cm}$ or we will do them in a planned staged program.

Despite these questions, there is little doubt that RFA for $\mathrm{BE}$ has dramatically altered the treatment paradigm for dysplastic $\mathrm{BE}$ and has resulted in the sparing of many an esophagus that would have otherwise been removed for this problem. The purpose of this study was to use endoscopic ablative technology in conjunction with laparoscopic ARS to improve electrode contact, thereby increasing the success rate of complete ablation. We show that this improves overall $\mathrm{BE}$ ablation efficiency, decreases BE recurrence, and will hopefully impact long-term cancer risk.

\section{CONCLUSIONS}

Intraoperative endoscopic RFA of BE at the time of laparoscopic fundoplication is feasible and might be a more efficient and cost-effective way to treat BE. We show that a single combined treatment results in the need for fewer overall procedures performed to obtain $\mathrm{BE}$ eradication. $\mathrm{Al}-$ though the complication rate of this pilot study was not negligible, patients did well with conservative treatment and our procedural approach has been subsequently modified with promising results. We believe that prospective study of this combined treatment modality for patients with $\mathrm{BE}$ is warranted.

\section{Author Contributions}

Study conception and design: Goers, Swanström.

Acquisition of data: Goers, Leão, Cassera.

Analysis and interpretation of data: Goers, Leão, Cassera, Swanström.

Drafting of manuscript: Goers, Cassera, Swanström.

Critical revision: Goers, Cassera, Dunst, Swanström.

\section{REFERENCES}

1. Sharma P, Dent J, Armstrong D, et al. The development and validation of an endoscopic grading system for Barrett's esophagus: the Prague C \& M criteria. Gastroenterology 2006;131: 1392-1399.
2. Sharma P, McQuaid K, Dent J, et al. A critical review of the diagnosis and management of Barrett's esophagus: the AGA Chicago Workshop. Gastroenterology 2004;127:310-330.

3. Wang KK, Sampliner RE. Updated guidelines 2008 for the diagnosis, surveillance and therapy of Barrett's esophagus. Am J Gastroenterol 2008;103:788-797.

4. Shaheen NJ, Sharma P, Overholt BF, et al. Radiofrequency ablation in Barrett's esophagus with dysplasia. N Engl J Med 2009; 360:2277-2288.

5. Spechler SJ. Clinical practice. Barrett's esophagus. N Engl J Med 2002;346:836-842.

6. Fleischer DE, Overholt BF, Sharma VK, et al. Endoscopic ablation of Barrett's esophagus: a multicenter study with 2.5-year follow-up. Gastrointest Endosc 2008;68:867-876.

7. Ganz RA, Utley DS, Stern RA, et al. Complete ablation of esophageal epithelium with a balloon-based bipolar electrode: a phased evaluation in the porcine and in the human esophagus. Gastrointest Endosc 2004;60:1002-1010.

8. Dunkin BJ, Martinez J, Bejarano PA, et al. Thin-layer ablation of human esophageal epithelium using a bipolar radiofrequency balloon device. Surg Endosc 2006;20:125-130.

9. Vassiliou MC, von Renteln D, Wiener DC, et al. Treatment of ultralong-segment Barrett's using focal and balloon-based radiofrequency ablation. Surg Endosc 2010;24:786-791.

10. Hubbard N, Velanovich V. Endoscopic endoluminal radiofrequency ablation of Barrett's esophagus in patients with fundoplications. Surg Endosc 2007;21:625-628.

11. dos Santos RS, Bizekis C, Ebright M, et al. Radiofrequency ablation for Barrett's esophagus and low-grade dysplasia in combination with an antireflux procedure: a new paradigm. JThorac Cardiovasc Surg 2010;139:713-716.

12. Smith CD, Bejarano PA, Melvin WS, et al. Endoscopic ablation of intestinal metaplasia containing high-grade dysplasia in esophagectomy patients using a balloon-based ablation system. Surg Endosc 2007;21:560-569.

13. Awad ZT, Mittal SK, Roth TA, et al. Esophageal shortening during the era of laparoscopic surgery. World J Surg 2001;25: $558-561$

14. Sharma VK, Wang KK, Overholt BF, et al. Balloon-based, circumferential, endoscopic radiofrequency ablation of Barrett's esophagus: 1-year follow-up of 100 patients. Gastrointest Endosc 2007;65:185-195.

15. Nicholson A, Jankowski J. Editorial: one small step for metaplasia, but one giant leap for biomarkers is needed. Am J Gastroenterol 2009; 104:2681-2683.

16. Vallbohmer D, Marjoram P, Kuramochi H, et al. Towards the molecular characterization of disease: comparison of molecular and histological analysis of esophageal epithelia. J Gastrointest Surg 2007;11:1095-1104.

17. Csendes A, Braghetto I, Burdiles P, et al. Late results of the surgical treatment of 125 patients with short-segment Barrett esophagus. Arch Surg 2009;144:921-927. 